\title{
Shape-Invariant Modeling of Circadian Rhythms with Random Effects and Smoothing Spline ANOVA Decompositions
}

\author{
Yuedong Wang, ${ }^{1, *}$ Chunlei Ke, ${ }^{2, * *}$ and Morton B. Brown,,${ }^{3, * * *}$ \\ ${ }^{1}$ Department of Statistics and Applied Probability, University of California, \\ Santa Barbara, California, U.S.A. \\ ${ }^{2}$ St. Jude Medical, Cardiac Rhythm Management Division, Sylmar, California, U.S.A. \\ ${ }^{3}$ Department of Biostatistics, University of Michigan, Ann Arbor, Michigan, U.S.A. \\ *email: yuedong@pstat.ucsb.edu \\ **email: cke@sjm.com \\ ***email: mbb@umich.edu
}

\begin{abstract}
Summary. Medical studies often collect physiological and/or psychological measurements over time from multiple subjects, to study dynamics such as circadian rhythms. Under the assumption that the expected response functions of all subjects are the same after shift and scale transformations, shapeinvariant models have been applied to analyze this kind of data. The shift and scale parameters provide efficient and interpretable data summaries, while the common shape function is usually modeled nonparametrically, to provide flexibility. However, due to the deterministic nature of the shift and scale parameters, potential correlations within a subject are ignored. Furthermore, the shape of the common function may depend on other factors, such as disease. In this article, we propose shape-invariant mixed effects models. A second-stage model with fixed and random effects is used to model individual shift and scale parameters. A second-stage smoothing spline ANOVA model is used to study potential covariate effects on the common shape function. We apply our methods to a real data set to investigate disease effects on circadian rhythms of cortisol, a hormone that is affected by stress. We find that patients with Cushing's syndrome lost circadian rhythms and their 24-hour means were elevated to very high levels. Patients with major depression had the same circadian shape and phases as normal subjects. However, their 24-hour mean levels were elevated and amplitudes were dampened for some patients.
\end{abstract}

KEY WORDS: Biological rhythm; Repeated measures data; Self-modeling nonlinear regression model; Semiparametric nonlinear mixed effects model.

\section{Introduction}

Biological rhythms are fundamental to living matter from subcellular particles to the human organism (Wever, 1979). For example, it is known that hormone cortisol levels peak in the morning and decrease to substantially lower levels late at night. Recent advances have shown the importance of these rhythms for everyday life, in health, as well as in disease (Kupfer, Monk, and Barchas, 1988; Reilly, Atkinson, and Waterhouse, 1997). Information on biological rhythms has been applied to almost every field of clinical and laboratory medicine (Hayes, Pauly, and Reiter, 1990; Touitou and Haus, 1992; Redfern and Lemmer, 1997).

Medical researchers are often interested in circadian rhythms: a cyclical variation in the intensity of a metabolic or physiological process with a period of about 24 hours. Experiments are typically conducted in such a way that variables of interest are measured several times during a time period, say, 24 hours, from a group of normal (or sick) human subjects (or animals). The problems of interest are: 1) do circadian rhythms exist? and 2) do demographic (e.g., age, sex), environmental (e.g., time-zone transition, workload), and medical, physiological or psychological (e.g., depression, stress) variables affect circadian rhythms, and if so, how?

In an experiment to study immunological responses in humans, blood samples were collected every two hours for 24 hours from 9 healthy normal volunteers, 11 patients with major depression and 16 patients with Cushing's syndrome. These blood samples were analyzed for parameters that measure immune functions and hormones of the hypothalamicpituitary-adrenal (HPA) axis (Kronfol et al., 1997). In this article, we will concentrate on cortisol. Figure 1 presents the observed concentrations (ug/dl) of the cortisol on a log scale from the nine healthy subjects.

It is well known that many hormones vary in a circadian pattern (Kronfol et al., 1997; Wang and Brown, 1996). Since the 24-hour periodicity is entrained, the cycle length is fixed. The common practice is to fit a single sinusoidal function to each subject (Monk, 1982; Cugini et al., 1990; Prins and 


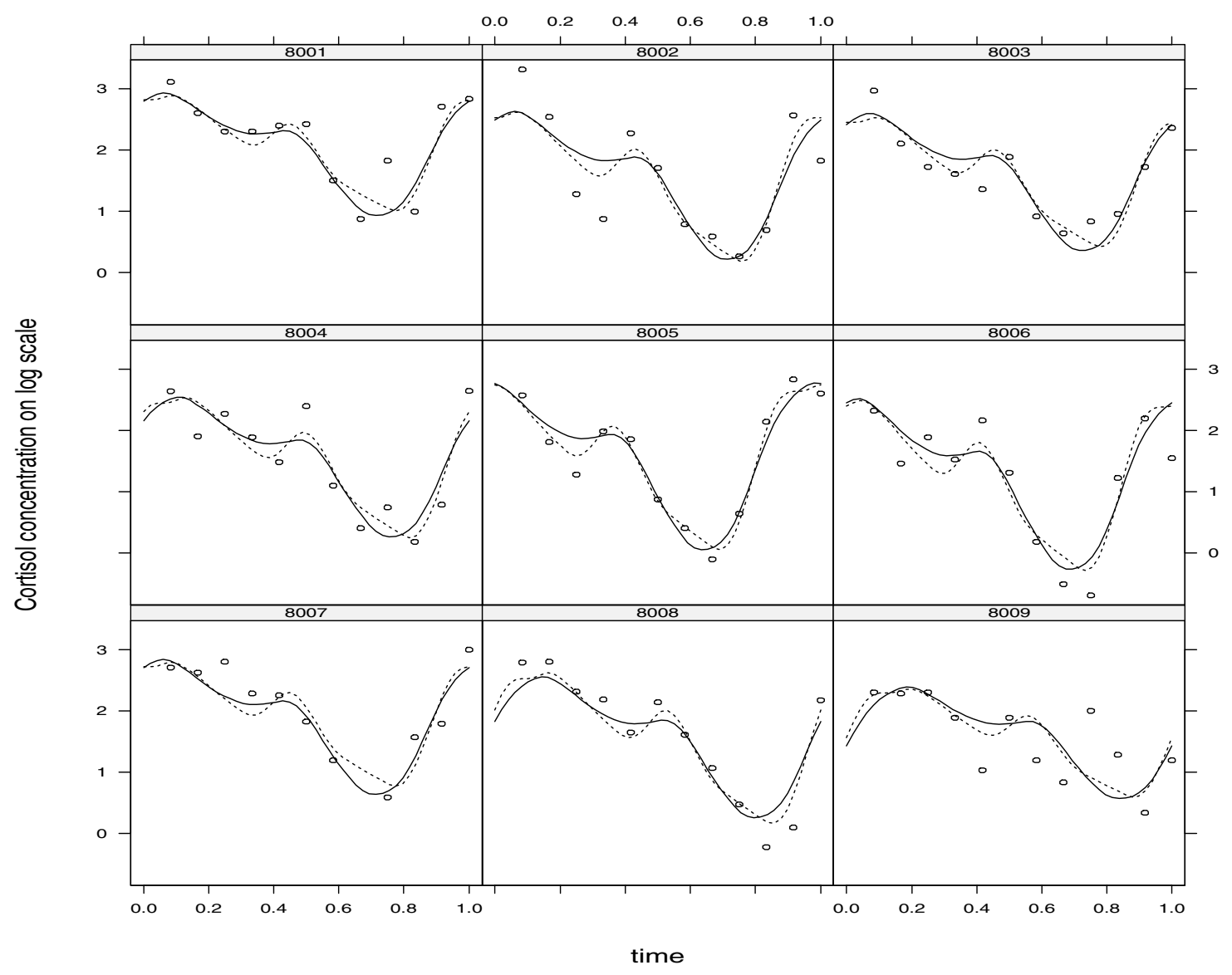

Figure 1. Plots of cortisol concentration on the log scale and fitted curves for normal subjects. Circles are observations. Solid lines represent fits from model (11). Dotted lines represent fits from model (8). Subjects' IDs are shown in the strip.

Hecquet, 1992). Problems with this approach are: (a) the pattern over time may not be symmetric; that is, the peak and nadir may not be separated by 12 hours and/or the amplitude and width of the peak may differ from those of the nadir, (b) sometimes there are local minimum and maximum points (Wang and Brown, 1996). It has been recognized that the sinusoidal function is too restrictive and "rhythms with a shape closely approximating a cosine curve are uncommon" (Reilly et al., 1997, p. 151). Thus, "other statistical methods must have preference which do not precondition any definite rhythm shape" (Wever, 1979, p. 20).

Although adding harmonics can improve the fit, it is difficult to decide how many harmonics to include in the model, and the results are difficult to interpret. From Figure 1, one can see that the data are noisy and it is difficult to identify patterns among subjects. Average measurements of all subjects are often plotted against time to find a common feature. Such a procedure may produce artifacts when subjects have different phases, means, or/and amplitudes (Reilly et al., 1997).

The goal of this article is to propose a new class of flexible methods and demonstrate their applications. In Section 2, we review the shape invariant models and introduce shape invariant mixed effects models. Analyses of the cortisol data are presented in Section 3. We conclude with discussions in Section 4 .

\section{Shape-Invariant Mixed Effects Models}

\subsection{Shape-Invariant Model for Circadian Rhythms}

Wang and Brown (1996) developed a flexible shape-invariant model (SIM) using a periodic spline function as the common curve, and modeled individual variations by 24-hour means, phases, and amplitudes. More precisely, they assumed that

$y_{i j}=\mu_{i}+\alpha_{i} f\left(t_{i j}-\tau_{i}\right)+\epsilon_{i j}, \quad i=1, \ldots, m ; \quad j=1, \ldots, n_{i}$,

where $m$ is the total number of subjects, $n_{i}$ is the number of observations for subject $i, y_{i j}$ is the response of $i$ th individual at the $j$ th time point $t_{i j}, \mu_{i}$ is the 24-hour mean of the $i$ th individual, $\alpha_{i}>0$ is the amplitude of the $i$ th individual, and $0 \leq \tau_{i}<1$ is the phase (horizontal shift) of the $i$ th individual. For simplicity, the 24-hour period is transformed into $[0,1]$. $\epsilon_{i j}$ 's are random errors and $\epsilon_{i j} \stackrel{i i d}{\sim} \mathrm{N}\left(0, \sigma^{2}\right)$.

The function $f$ in (1) is the common circadian shape function. It is a periodic function with period one. For 
identifiability of model (1), it is also assumed that the integral of $f$ equals zero and $\sup _{t \in[0,1]}|f(t)|=1$. Specifically, let

$$
\begin{aligned}
& W_{2}^{0}(\text { per })=\left\{f: f \text { and } f^{\prime}\right. \text { are absolutely continuous, } \\
& \int_{0}^{1}\left(f^{\prime \prime}(t)\right)^{2} d t<\infty, \int_{0}^{1} f(t) d t=0, f(0)=f(1) \\
&\left.f^{\prime}(0)=f^{\prime}(1)\right\}
\end{aligned}
$$

be the reproducing kernel Hilbert space of cubic splines on the circle, without the constant term (Wahba, 1990). The model space for $f$ in (1) is

$$
\mathcal{M}=\left\{f \in W_{2}^{0}(\text { per }): \sup _{t \in[0,1]}|f(t)|=1\right\} .
$$

The SIMs have also been applied to areas such as human growth (Stutzle et al., 1980), spirometry, spectrophometric, and sensitometric analyses (Lawton, Sylvestre, and Maggio, 1972), bioassay (Guardabasso, Rodbard, and Munson, 1987), curve registration (Ramsay and $\mathrm{Li}, 1998$ ), and metabolism (Altman, 1996). The advantage of model (1) is that no specific form has been assumed for the common function; it is only assumed to be periodic and smooth, and its shape is decided by the data. Therefore, the model is very flexible. Note that the sinusoidal function is a special case of model (1), with $f(t)=\sin 2 \pi t$. The periodic spline function in model (1) reduces to a sinusoidal function if the extra flexibility is not warranted by data. Observations will be pooled from all subjects to estimate this shape function. Parameters of interest to scientists (24-hour mean, amplitude, and phase) remain the same as those in a sinusoidal model. However, model (1) has the following drawbacks: (a) the parameters $\mu_{i}, \alpha_{i}$ and $\tau_{i}$ in model (1) were assumed to be fixed effects. As a consequence, observations from the same subject were assumed to be independent; (b) besides the nonparametric function $f$, the number of parameters equals $3 m+1$, which increases with the number of subjects. This may adversely affect the estimation and inference for the parameters under the common experimental situation that the number of subjects is large and the number of observations for each subject is small; (c) it is difficult to investigate covariate effects on parameters and/or the common curve. Ad hoc second-stage analyses are often used to investigate covariate effects. In the next section, we use mixed effects models for the parameters and smoothing spline ANOVA models for the common curve to overcome these problems.

\subsection{Shape-Invariant Mixed Effects Models}

We will construct a two-stage model. At the first stage, we assume the same model as (1) and rewrite it as

$$
\begin{array}{r}
y_{i j}=\phi_{1 i}+\exp \left(\phi_{2 i}\right) f\left(t_{i j}-\operatorname{alogit}\left(\phi_{3 i}\right)\right)+\epsilon_{i j}, \\
i=1, \ldots, m, \quad j=1, \ldots, n_{i} .
\end{array}
$$

Note that the exponential transformation is used to force the amplitude to be positive and the inverse logistic transformation, $\operatorname{alogit}(x)=\exp (x) /(1+\exp (x))$, is used to force the phase to be inside the interval $[0,1]$. We will allow random errors within a subject to be correlated: $\boldsymbol{\epsilon}_{i}=\left(\epsilon_{i 1}, \ldots, \epsilon_{i n_{i}}\right)^{T} \sim$ $N\left(0, \sigma^{2} \boldsymbol{\Lambda}_{i}\right)$. Thus, model (4) itself is more general than the classical SIM (1).

Let $\phi_{i}=\left(\phi_{1 i}, \phi_{2 i}, \phi_{3 i}\right)^{T}$. Denote $\boldsymbol{z}_{i}$ as the covariate vector of subject $i$. At the second stage, between-subject differences are modeled using covariate information with the following linear model (Lindstrom and Bates, 1990):

$$
\boldsymbol{\phi}_{i}=\boldsymbol{A}_{i} \boldsymbol{\beta}+\boldsymbol{B}_{i} \boldsymbol{b}_{i}, \quad \boldsymbol{b}_{i} \stackrel{i i d}{\sim} \mathrm{N}\left(0, \sigma^{2} \boldsymbol{D}\right)
$$

where $\boldsymbol{\beta}$ is a $p$-vector of fixed population parameters, $\boldsymbol{b}_{i}$ is a $q$-vector of random effects associated with subject $i$, and $\boldsymbol{A}_{i}$ and $\boldsymbol{B}_{i}$ are $3 \times p$ and $3 \times q$ design matrices determined by the covariate vector $\boldsymbol{z}_{i}$. More complicated models, such as the nested model in Section 3, may be used.

Covariate effects on the circadian shape function can be modeled using a smoothing spline ANOVA (SS ANOVA) decomposition

$$
f(\boldsymbol{z}, t)=\mu+f_{1}(\boldsymbol{z})+f_{2}(t)+f_{12}(\boldsymbol{z}, t),
$$

where $\mu$ is a constant, $f_{1}$ and $f_{2}$ are the main effects of covariates $\boldsymbol{z}$ and $t$, respectively, and $f_{12}$ is the interaction between $\boldsymbol{z}$ and $t$. Note that $\boldsymbol{z}$ could be a vector of covariates. Thus, further SS ANOVA decompositions may be constructed for $f_{1}$ and $f_{12}$. What model space and SS ANOVA decomposition to use for $f$ depends on the domains of $\boldsymbol{z}$ and $t$, prior knowledge, constraints for identifiability, and purpose of the analysis. See Wahba (1990) and Gu (2002) for details about SS ANOVA decompositions.

Equations (4), (5), and (6) altogether define a shapeinvariant mixed effects model (SIMM). Interpretations of the parameters remain the same. Correlation within a subject is modeled with random effects and random errors. Covariate effects on parameters and/or the common curve are part of the model. The awkward constraint for identifiability, $\sup _{t \in[0,1]}|f(t)|=1$, can be dropped by removing a constant term from the fixed effect of $\phi_{2 i}$.

Lindstrom (1995) extended the SIM by including random shift and scale parameters. She used a free-knot spline with a fixed number of knots to model the common curve. Our second-stage models are more general. The second-stage SS ANOVA model (6) for the common function is especially new. Our procedure allows data to mold the shape of the common curve. For simplicity, our methods are presented using periodic splines. It is straightforward to generalize these methods to the setting of general smoothing splines (Wahba, 1990).

\subsection{Estimation, Inference, and Software}

Because $f$ interacts with parameters (fixed or random) in a nonlinear manner, estimation is complicated. Ke and Wang (2001) developed estimation methods for semiparametric nonlinear mixed effects models (SNM). Since the SIMM is a special case of the SNM model, the methods developed for SNM models can be used.

We now briefly describe these methods for SIMMs. Consider a SIMM (4) with an SS ANOVA model (6) for $f$ and a second-stage model (5) for $\phi_{i}$ 's. Let a vector $\boldsymbol{\theta}$ contain all parameters in $\boldsymbol{\Lambda}_{i}$ 's and $\boldsymbol{D}$. We need to estimate parameters $\boldsymbol{\beta}$, 
$\sigma^{2}$, and $\boldsymbol{\theta}$, the nonparametric function $f$ and its components $\mu, f_{1}, f_{2}$, and $f_{12}$, and random effects $\boldsymbol{b}_{i}$ 's. Let $N=\sum_{i=1}^{m} n_{i}$, $\boldsymbol{y}_{i}=\left(y_{i 1}, \ldots, y_{i n_{i}}\right)^{T}, \eta\left(\phi_{i}, f ; \boldsymbol{z}_{i}, t_{i j}\right)=\phi_{1 i}+\exp \left(\phi_{2 i}\right)$ $f\left(\boldsymbol{z}_{i}, t_{i j}-\operatorname{alogit}\left(\phi_{3 i}\right)\right)$, and $\boldsymbol{\eta}_{i}\left(\phi_{i}, f\right)=\left(\eta\left(\phi_{i}, f ; \boldsymbol{z}_{i}, t_{i 1}\right), \ldots\right.$, $\left.\eta\left(\boldsymbol{\phi}_{i}, f ; \boldsymbol{z}_{i}, t_{i n_{i}}\right)\right)^{T}$. Let

$$
\begin{aligned}
L\left(\boldsymbol{\beta}, f, \boldsymbol{\theta}, \sigma^{2}\right)= & \left(2 \pi \sigma^{2}\right)^{-\frac{m q+N}{2}}|\boldsymbol{D}|^{-\frac{m}{2}}\left(\prod_{i=1}^{m}\left|\boldsymbol{\Lambda}_{i}\right|^{-\frac{1}{2}}\right) \\
& \times \int \exp \left[-g(\boldsymbol{b}) / \sigma^{2}\right] d \boldsymbol{b},
\end{aligned}
$$

be the marginal likelihood based on the SIMM, where $\boldsymbol{b}=$ $\left(\boldsymbol{b}_{1}^{T}, \ldots, \boldsymbol{b}_{m}^{T}\right)^{T}$, and

$$
\begin{aligned}
g(\boldsymbol{b})= & \frac{1}{2} \sum_{i=1}^{m}\left[\left(\boldsymbol{y}_{i}-\boldsymbol{\eta}\left(\boldsymbol{A}_{i} \boldsymbol{\beta}+\boldsymbol{B}_{i} \boldsymbol{b}_{i}, f\right)\right)^{T} \boldsymbol{\Lambda}_{i}^{-1}\right. \\
& \left.\times\left(\boldsymbol{y}_{i}-\boldsymbol{\eta}\left(\boldsymbol{A}_{i} \boldsymbol{\beta}+\boldsymbol{B}_{i} \boldsymbol{b}_{i}, f\right)\right)+\boldsymbol{b}_{i}^{T} \boldsymbol{D}^{-1} \boldsymbol{b}_{i}\right] .
\end{aligned}
$$

Ke and Wang (2001) used Laplace's method to approximate the log likelihood $l\left(\boldsymbol{\beta}, f, \boldsymbol{\theta}, \sigma^{2}\right)=\log \left(L\left(\boldsymbol{\beta}, f, \boldsymbol{\theta}, \sigma^{2}\right)\right)$, because the integral with respect to the random effects $\boldsymbol{b}$ is usually intractable. For fixed $\boldsymbol{\beta}, f, \boldsymbol{\theta}$, and $\sigma^{2}$, we estimate random effects $\boldsymbol{b}$ by the minimizer $\tilde{\boldsymbol{b}}$ of $g(\boldsymbol{b})$. Applying Laplace's method for integral approximation, we approximate $l\left(\boldsymbol{\beta}, f, \boldsymbol{\theta}, \sigma^{2}\right)$ by

$$
\begin{aligned}
\tilde{l}\left(\boldsymbol{\beta}, f, \boldsymbol{\theta}, \sigma^{2}\right) \\
=-\frac{N}{2} \log 2 \pi \sigma^{2}-\frac{1}{2} \sum_{i=1}^{m}\left(\log \left|\boldsymbol{\Lambda}_{i}\right|+\log \left|\boldsymbol{I}+\tilde{\boldsymbol{Z}}_{i}^{T} \boldsymbol{\Lambda}_{i}^{-1} \tilde{\boldsymbol{Z}}_{i} \boldsymbol{D}\right|\right) \\
\quad-\frac{1}{2 \sigma^{2}} \sum_{i=1}^{m}\left[\left(\boldsymbol{y}_{i}-\boldsymbol{\eta}_{i}\left(\boldsymbol{A}_{i} \boldsymbol{\beta}+\boldsymbol{B}_{i} \tilde{\boldsymbol{b}}_{i}, f\right)\right)^{T} \boldsymbol{\Lambda}_{i}^{-1}\right. \\
\left.\quad \times\left(\boldsymbol{y}_{i}-\boldsymbol{\eta}_{i}\left(\boldsymbol{A}_{i} \boldsymbol{\beta}+\boldsymbol{B}_{i} \tilde{\boldsymbol{b}}_{i}, f\right)\right)+\tilde{\boldsymbol{b}}_{i}^{T} \boldsymbol{D}^{-1} \tilde{\boldsymbol{b}}_{i}\right]
\end{aligned}
$$

where $\tilde{\boldsymbol{Z}}_{i}=\partial \boldsymbol{\eta}_{i}\left(\boldsymbol{A}_{i} \boldsymbol{\beta}+\boldsymbol{B}_{i} \boldsymbol{b}_{i}, f\right) /\left.\partial \boldsymbol{b}_{i}^{T}\right|_{\boldsymbol{b}_{i}=\boldsymbol{b}_{i}}$. For fixed $\tilde{\boldsymbol{b}}, \sigma^{2}$, and $\boldsymbol{\theta}$, we estimate $\boldsymbol{\beta}$ and $f$ as minimizers of the approximate penalized log likelihood

$$
-\tilde{l}\left(\boldsymbol{\beta}, f, \boldsymbol{\theta}, \sigma^{2}\right)+N \lambda_{1} J_{1}\left(f_{1}\right)+N \lambda_{2} J_{2}\left(f_{2}\right)+N \lambda_{12} J_{12}\left(f_{12}\right),
$$

where $J_{1}\left(f_{1}\right), J_{2}\left(f_{2}\right)$, and $J_{12}\left(f_{12}\right)$ are penalties for nonparametric components in (6), and $\lambda_{1}, \lambda_{2}$, and $\lambda_{12}$ are smoothing parameters. Denoting the solutions to $(7)$ as $\tilde{\boldsymbol{\beta}}$ and $\tilde{f}$, and plugging them into the approximate log likelihood, we then estimate $\sigma^{2}$ and $\boldsymbol{\theta}$ as the maximizers to the profile $\log$ likelihood $\tilde{l}\left(\tilde{\boldsymbol{\beta}}, \tilde{f}, \boldsymbol{\theta}, \sigma^{2}\right)$. Estimates of $\boldsymbol{b}, \boldsymbol{\beta}, f, \boldsymbol{\theta}$, and $\sigma^{2}$ can be calculated by iterating the above process until convergence. Further simplifications for each step can be found in Ke and Wang (2001). Data-adaptive criteria, such as generalized cross-validation, unbiased risk, and restricted maximum likelihood, are used to estimate the smoothing parameters (Ke and Wang, 2001; Wang, 1998).

Wang and Ke (2002) developed a user-friendly S-Plus package, ASSIST, for fitting many spline based models. One function, snm, is designed for fitting general SNM models. Thus, this function can be used to fit SIMMs. This package is available at http: //www.pstat.ucsb.edu/faculty/yuedong/ research. Programs used for fitting the hormone data are shown in the manual of this package.
Exact inference is difficult, due to the complicated relationship between $f$ and parameters. We use conditional inference. Inference for $f$ conditional on the estimated parameters was described in Ke and Wang (2001). Inference for parameters conditional on the estimate of $f$ can be conducted as follows: when $f$ is fixed, the SIMM reduces to a nonlinear mixed effects model. Thus, standard methods such as the t-test based on equation (7.20) in Pinheiro and Bates (2000) can be used. The residual degrees of freedom are adjusted to reflect the cost for estimating $f$.

\section{Data Analyses}

The data set obtained from the experiment described in Section 1 contains the following variables: $I D$ of subjects, time when measurements were taken, cortisol concentrations on log scale (named as horm), and subject group (normal, depression, Cushing's syndrome). For simplicity, the time variable of a 24 -hour period is transformed into $[0,1]$. Observations are shown in Figures 1, 2, and 3 as circles.

We first fit the following SIMM for each group

$$
\begin{array}{r}
y_{i j}=\beta+b_{1 i}+\exp \left(b_{2 i}\right) f\left(t_{i j}-\operatorname{alogit}\left(b_{3 i}\right)\right)+\epsilon_{i j}, \\
i=1, \ldots, m, \quad j=1, \ldots, n_{i},
\end{array}
$$

where the fixed effect $\beta$ represents 24-hour mean of the population, the random effects $b_{1 i}, b_{2 i}$, and $b_{3 i}$ represent the $i$ th subject's deviation of 24-hour mean, amplitude, and phase. We assume that $f \in W_{2}^{0}($ per $)$ and $\boldsymbol{b}_{i}=\left(b_{1 i}, b_{2 i}, b_{3 i}\right)^{T} \stackrel{i i d}{\sim}$ $N\left(0, \sigma^{2} \boldsymbol{D}\right)$, where $\boldsymbol{D}$ is an unstructured positive-definite matrix. The assumption of zero population mean for amplitude and phase parameters takes care of potential confounding between amplitude, phase, and the nonparametric common function $f$ in a natural way. In terms of notation in model (5), we have $\boldsymbol{A}_{i}=(1,0,0)^{T}$ and $\boldsymbol{B}_{i}$ is a $3 \times 3$ identity matrix.

The fits are shown in Figures 1, 2, and 3 as dotted lines. The estimated common functions are shown in Figure 4, together with their 95\% Bayesian confidence intervals.

Observations close in time from the same subject may be correlated. We fit model (8), with random errors within each subject modeled by a first-order autoregressive structure. The estimated lag 1 autocorrelation coefficients are small. Estimates of other parameters remain the same. Therefore, random errors are assumed to be independent in the remaining of this section.

From Figures 3 and 4, it is apparent that the common function for the Cushing's syndrome group is almost zero, which indicates that circadian rhythms were lost for patients with Cushing's syndrome. The absence of a circadian rhythm has been considered as hallmark of the diagnosis of Cushing's syndrome (Boyar et al., 1979; Liu, Kazer, and Rasmussen, 1987). Unlike normal subjects, patients with Cushing's syndrome fail to decrease cortisol secretion in the late evening. Therefore, the measurement of elevated late-evening cortisol is a very simple and useful way to screen patients for Cushing's syndrome (Raff, Raff, and Findling, 1998; Castro, Elias, and Quidute, 1999). It has also been noticed that some patients with Cushing's syndrome still demonstrate circadian rhythms (Refetoff et al., 1985; Tourniaire, Chalendar, and Rebbatu, 1986). Figure 3 suggests that cortisol levels of patients 3044 and 3069 may still have circadian rhythms. 


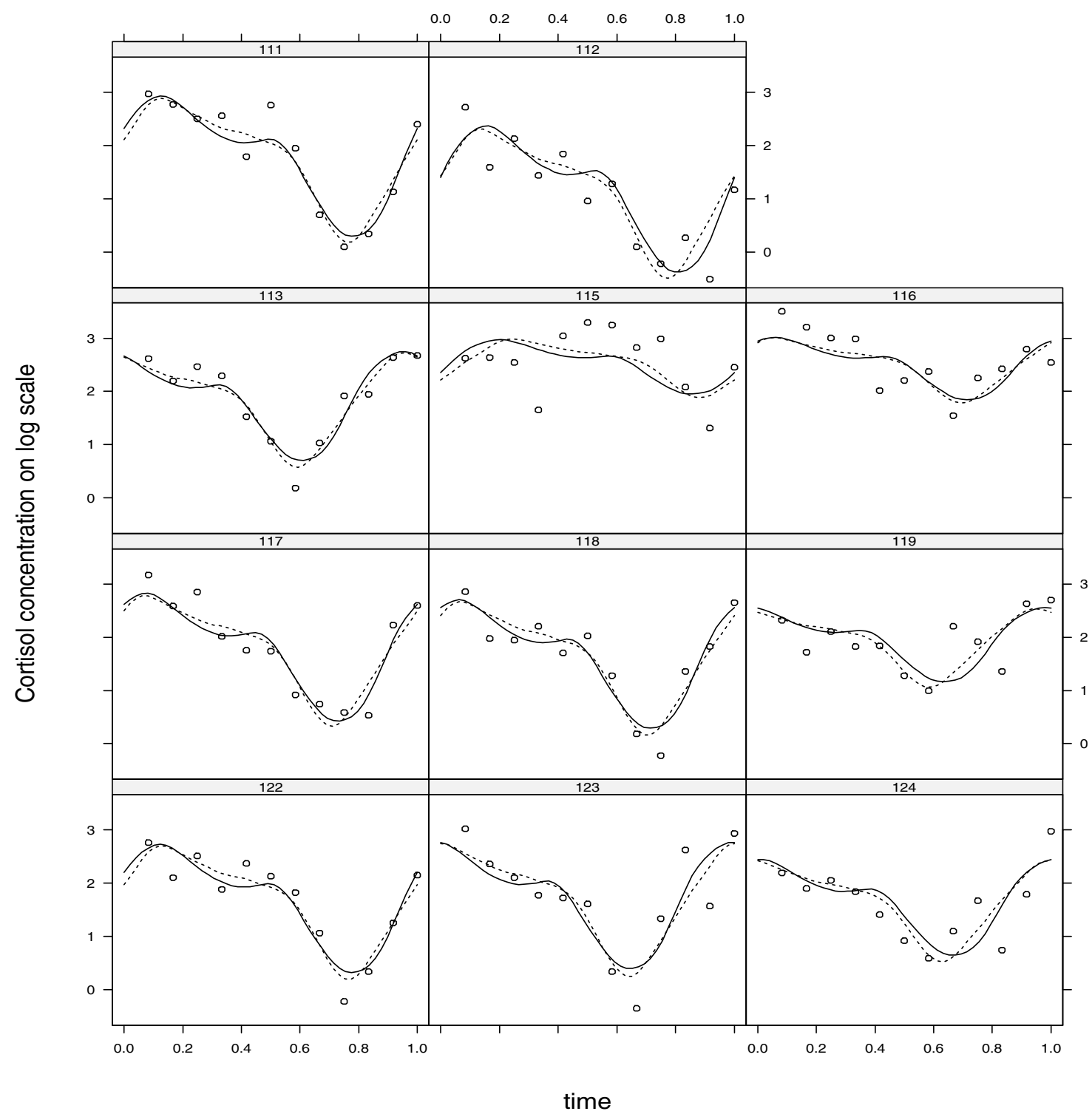

Figure 2. Plots of cortisol concentration on the log scale and fitted curves for patients with major depression. Circles are observations. Solid lines represent fits from model (11). Dotted lines represent fits from model (8). Subjects' IDs are shown in the strip.

We now compare patients having major depression with normal subjects. We will first investigate potential effects of depression on the shape function. We find that these two groups had the same shape function, which allows us to further investigate potential effects of depression on the parameters.

The shape functions for the normal and depression groups are similar (Figure 4). We now test the hypothesis that the shape functions of these two groups are equal. We achieve this by fitting data from these two groups simultaneously. Consider the following model

$$
\begin{array}{r}
y_{i j k}=\beta_{k}+b_{1 i(k)}+\exp \left(b_{2 i(k)}\right) f\left(k, t_{i j k}-\operatorname{alogit}\left(b_{3 i(k)}\right)\right)+\epsilon_{i j k}, \\
i=1, \ldots, m, \quad j=1, \ldots, n_{i k}, \quad k=1,2, \quad(9)
\end{array}
$$

where $k$ represents group with $k=1$ and $k=2$ corresponding to depression and normal groups, respectively, fixed effect $\beta_{k}$ is the population 24-hour mean of group $k$, random effects $b_{1 i(k)}, b_{2 i(k)}$, and $b_{3 i(k)}$ represent the ith subject's deviation of 24-hour mean, amplitude and phase. Note that subjects are nested within group, which is reflected in our notations. We allow different correlation structures for the random effects in each group. That is, we assume that $\boldsymbol{b}_{i(k)}=$ $\left(b_{1 i(k)}, b_{2 i(k)}, b_{3 i(k)}\right) \stackrel{\text { Tid }}{\sim} N\left(0, \sigma^{2} \boldsymbol{D}_{k}\right)$, where the $\boldsymbol{D}_{k}$ 's are unstructured positive-definite matrices. We assume different common functions for each group. Thus, $f$ is a function of both group (denoted as $k$ ) and time (denoted as $t$ ). We model the group effect using a one-way ANOVA model with model space $R^{2}$ and the time effect, using a periodic spline model with model space $W_{2}^{0}(p e r)$, where $R^{2}$ is the Euclidean 


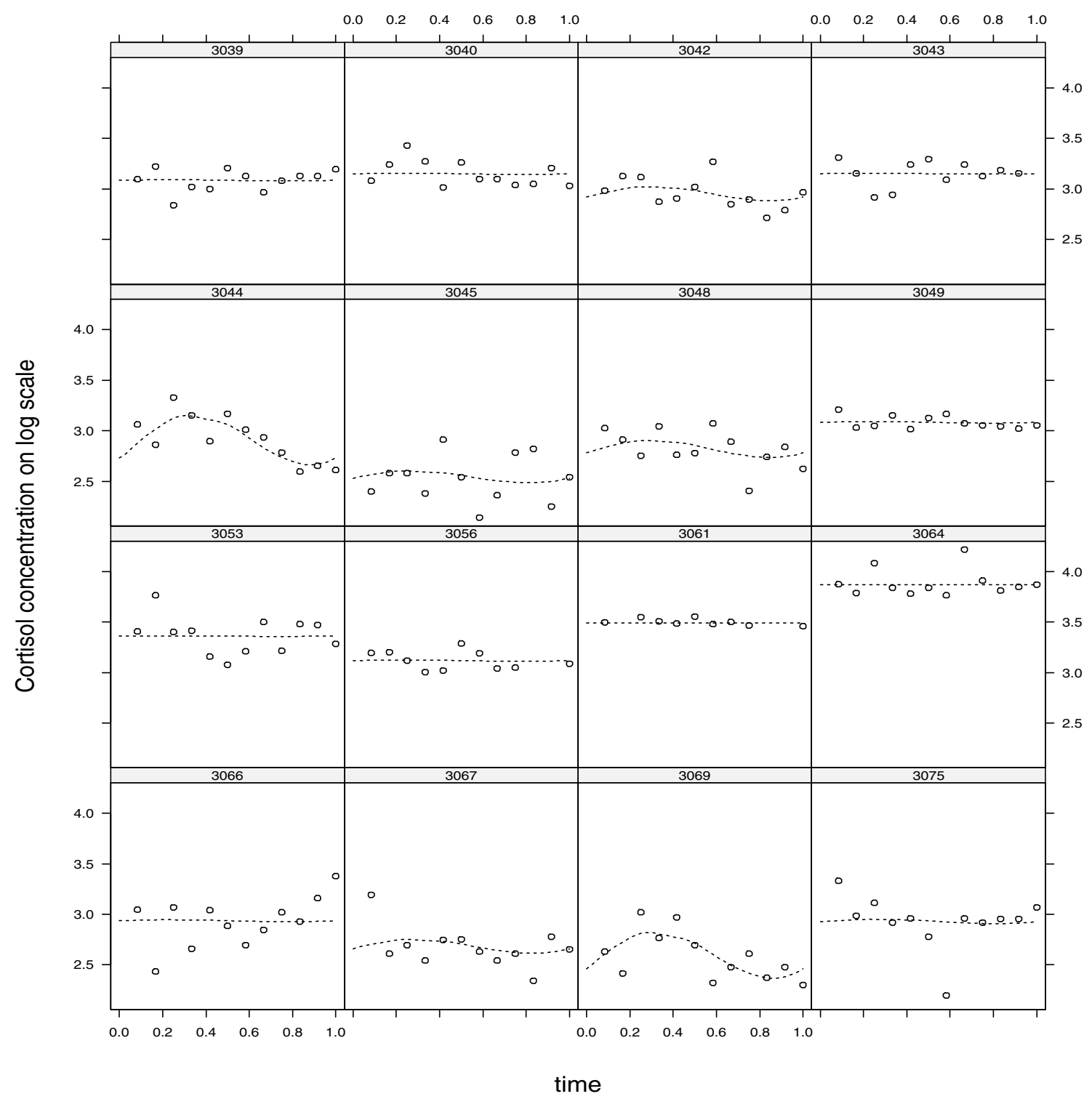

Figure 3. Plots of cortisol concentration on the log scale and fitted curves for patients with Cushing's syndrome. Circles are observations. Dotted lines represent fits from model (8). Subjects' IDs are shown in the strip.

two-space. That is, we assume that $f \in R^{2} \otimes W_{2}^{0}($ per $)$. Writing $R^{2}=\{1\} \oplus\left\{g: \sum_{k=1}^{2} g(k)=0\right\}$, where $\{1\}=\{g: g(1)=g(2)\}$ is the subspace of constant functions, we have the following SS ANOVA decomposition

$R^{2} \otimes W_{2}^{0}($ per $)=W_{2}^{0}($ per $) \oplus\left[\left\{g: \sum_{k=1}^{2} g(k)=0\right\} \otimes W_{2}^{0}(\right.$ per $\left.)\right]$,

or equivalently,

$$
f(k, t)=s(t)+s s(k, t)
$$

where $s(t)$ is the main effect of time, and $s s(k, t)$ is the interaction between group and time. It is easy to see that the hypothesis $H_{0}: f(1, t)=f(2, t)$ is equivalent to $H_{0}: s s(k, t)=0$.
The estimated smoothing parameter for the interaction term $s s(k, t)$ is large, which means that the interaction is small. In fact, $s s(k, t)$ is essentially zero: the estimates are on the magnitude of $10^{-6}$, while the posterior standard deviations are on the magnitude of $10^{-4}$. Therefore, we conclude that normal subjects and patients with major depression have the same shape function. This result confirms the assumption made in Wong et al. (2000), which was based on plots rather than on a formal test. Note that the classical methods based on a sinusoidal function implicitly assume the same shape functions for different groups. Thus, these methods cannot be used to test such a hypothesis, and there is no test available in the literature.

Under the assumption of one shape function for both groups, we now can investigate differences of 24-hour mean, amplitude, and phase between these two groups. For this 


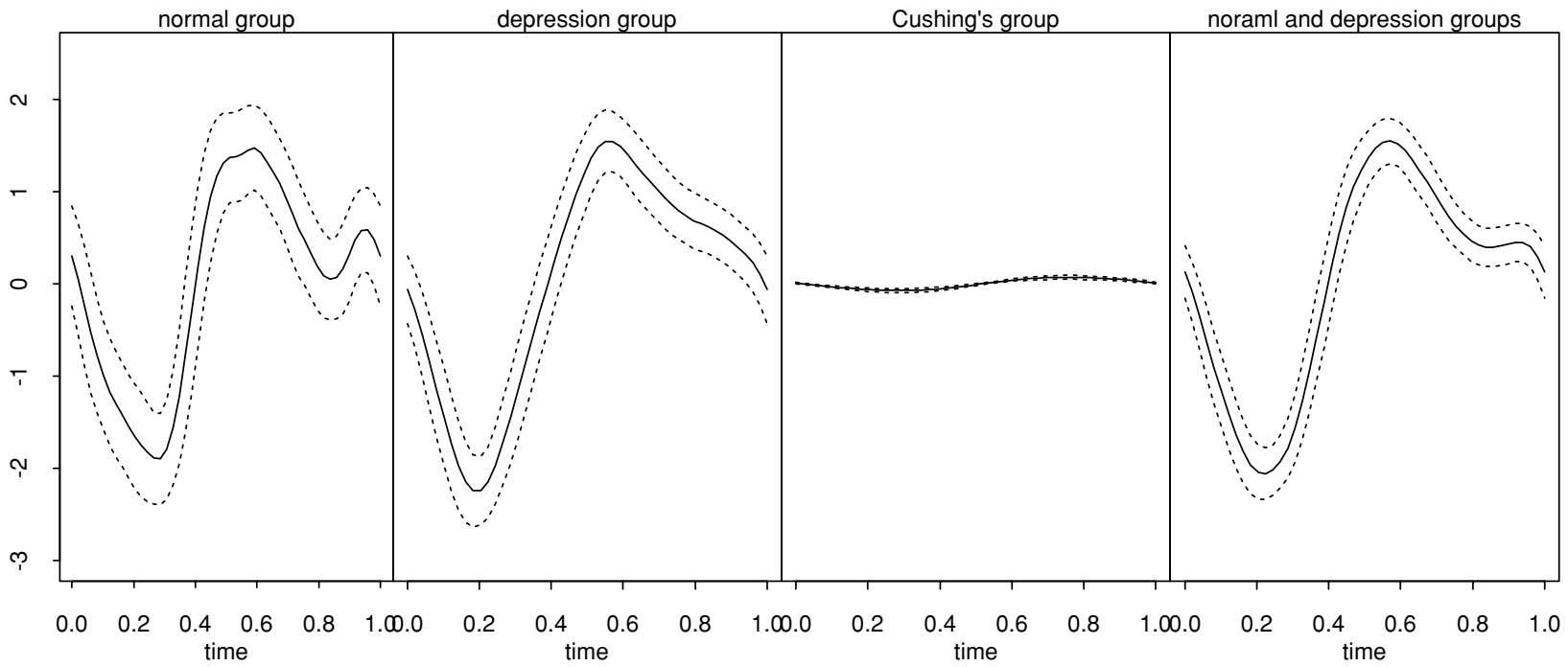

Figure 4. Solid lines are estimates of the common functions and dotted lines are 95\% Bayesian confidence intervals. The left three panels are estimated common curves for the normal, depression, and Cushing's syndrome groups respectively. The right panel is the estimated common curve in model (11).

purpose, we consider the following model

$$
\begin{aligned}
y_{i j k}= & \beta_{k}+b_{1 i(k)}+\exp \left(b_{2 i(k)}+d_{1} \times I_{[k=2]}\right) \\
\times & f\left(t_{i j k}-\operatorname{alogit}\left(b_{3 i(k)}+d_{2} \times I_{[k=2]}\right)\right)+\epsilon_{i j k}, \\
& i=1, \ldots, m, \quad j=1, \ldots, n_{i k}, k=1,2,
\end{aligned}
$$

where $\beta_{2}-\beta_{1}, d_{1}$ and $d_{2}$ measure the differences of 24-hour mean, amplitude and phase between the normal group and the depression group.

Estimates of $\beta_{2}-\beta_{1}, d_{1}$ and $d_{2}$ are $-0.2724(\mathrm{SE}=0.1311$, $\mathrm{p}$-value $=0.0389), 0.2350(\mathrm{SE}=0.0767, \mathrm{p}$-value $=0.0024)$ and $0.0299(\mathrm{SE}=0.0916, \quad \mathrm{p}$-value $=0.7441)$, respectively. Standarded errors and p-values are calculated based on the ttest described in Section 2. We conclude that the differences of 24-hour mean and amplitude are significant, while the difference of phase is not. We refit model (11) without the $d_{2}$ term. The fits are shown in Figures 1 and 2 as solid lines.
The right panel of Figure 4 shows the estimated common function and its 95\% Bayesian confidence intervals from the final model. Data from these two groups are pooled to estimate the common function. Thus, the Bayesian confidence intervals are narrower.

Figure 5 shows the estimated 24-hour mean levels plotted against the estimated amplitudes. The amplitudes for patients with Cushing's syndrome are adjusted to have the same scale as those for normal subjects and patients with major depression. We conclude that for patients with Cushing's syndrome, 24-hour means were elevated to much higher levels. Circadian rhythms were lost except for patients 3044 and 3069. For patients with major depression, 24-hour means were elevated. However, their amplitudes were similar to those of normal subjects, except for patients 115 and 116, whose 24-hour mean levels and amplitudes were closer to those of patients with Cushing's syndrome. The statistically significant differences of

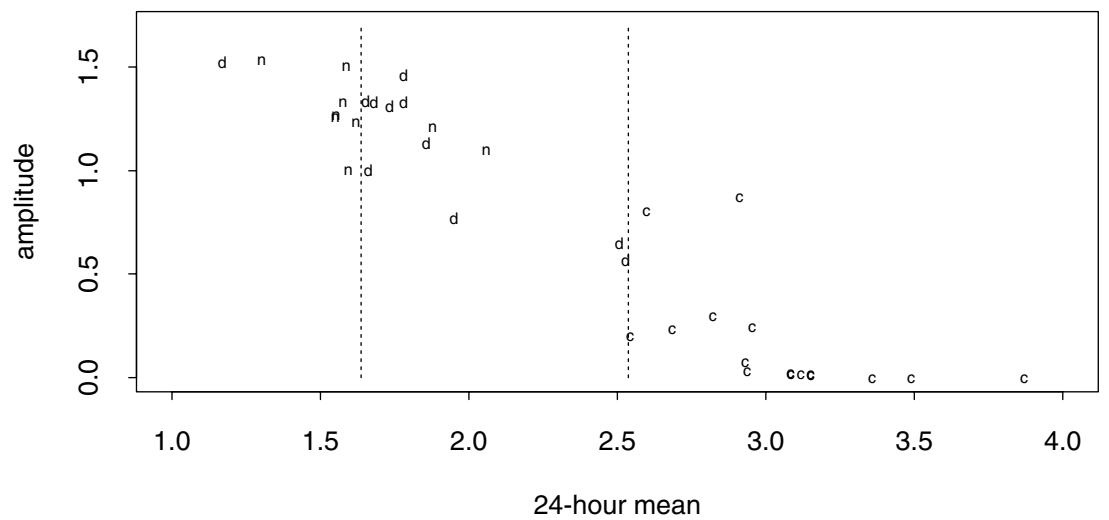

Figure 5. Plot of the estimated 24-hour mean levels against amplitudes. Normal subjects, patients with major depression, and patients with Cushing's syndrome are marked as n, d, and c, respectively. Dotted lines represent partition of three groups based on the tree method. 
the amplitudes between the normal group and the depression group are mainly caused by these two patients. The highly elevated cortisol levels and waning circadian rhythms in these two patients may suggest that their conditions were worse, and that further medical tests are necessary to check if they have Cushing's syndrome.

Based on a different study, Wong et al. (2000) concluded that 30-hour mean cortisol levels were significantly elevated in patients with melancholic depression. They did not compare the differences of amplitudes. Our results are consistent with those in Wong et al. (2000).

Figure 5 shows a negative relationship between the 24-hour mean and amplitude. The estimated correlations between $b_{1 i(k)}$ and $b_{2 i(k)}$ are -0.786 for the depression group $(k=1)$ and -0.531 for the normal group $(k=2)$. This indicates that the negative correlation between the 24-hour mean and the amplitude exists not only at the group level, but also at the subject level. The difference in correlations may be used to investigate physiological changes (Tsaneva, Topalova, and Beraha, 1990).

\section{Discussion}

This article presents a flexible and coherent approach to investigate covariate effects on circadian rhythms. As in the classical ANOVA, we decompose the multivariate function $f(k, t)$ into its main effects and interactions. The original hypothesis is transformed into one on the interaction component. In general, this technique can be used to test differences between two functions (Härdle and Marron, 1990; Wang and Ke, 2002). The second stage SS ANOVA model (6) is defined for general $\boldsymbol{z}$. For example, letting $\boldsymbol{z}=$ (gender, age), we can investigate gender and age effects on circadian rhythms (Cauter, Leproult, and Kupfer, 1996). For our specific application, we concentrated on circadian rhythms. The models and methods developed in this article can be applied to ultradian and infradian rhythms too. If the period is not fixed and unknown, a scale parameter can be added to model (4).

It is noted that symptoms suggesting the presence of Cushing's syndrome are not pathognomonic. Thus, diagnosis may be nonspecific (e.g., obesity, hypertension, menstrual irregularity, and glucose intolerance; Orth, 1995). Plots such as Figure 5 may be helpful in improving the precision of diagnosis and/or to further cluster patients into groups. A simple classification by the tree method (Breiman et al., 1984) is presented in Figure 5 as dotted lines. It uses the 24-hour mean only, and classifies a subject as normal, depression, and Cushing's syndrome if his 24-hour mean is below 1.64, above 1.64 but below 1.87, and above 1.87 , respectively.

Besides cortisol, the experiment also measured many other variables, such as CD4 and the hormone ACTH. The methods in this article can be used to investigate disease effects on circadian rhythms for all these variables. It can also be used to investigate the possible association between variables such as ACTH and cortisol (Wang, Guo, and Brown, 2000), and disease effects on the association (Wong et al., 2000).

\section{ACKNOWLEDGEMENTS}

The authors are grateful to Ziad Kronfol, MD, from the University of Michigan Medical School, for permission to use these data. Support for this research was provided by the National Institute of Health under grants R01 GM58533, P60 DK20572, and M01 RR00042. The authors thank the editor, the associate editor, and referee for their constructive comments and suggestions that have led to a substantial improvement in the article.

\section{RÉSUMÉ}

Des études médicales recueillent souvent des mesures physiologiques et/ou psychologiques au cours du temps à partir de nombreux de sujets dans le but d'étudier la dynamique tel que les rythmes circadiens. Sous l'hypothèse que les fonctions de réponse attendues pour tous les sujets sont les mêmes après les décalages et transformations d'échelle, les modèles de forme invariante ont été utilisé pour analyser ce type de données. Le décalage et les paramètres d'échelle sont des résumés des données efficaces et interprétables, tandis que la fonction de forme commune est modélisée classiquement de manière non paramétrique pour fournir de la flexibilité. Cependant, en raison de la nature déterministe du décalage et des paramètres d'échelle, les corrélations potentielles intra-sujet sont ignorées. En outre, la forme de la fonction commune peut dépendre d'autres facteurs comme la maladie. Dans ce papier, nous proposons la forme des modèles à effets mixtes de forme invariante. Un modèle à 2 étapes à effets fixes et aléatoires est utilisé pour modéliser le décalage individuel et les paramètres d'échelle. Une deuxième étape utilise un modèle d'ANOVA de lissage par splines pour étudier l'effet potentiel de covariables sur la fonction de forme commune. Nos méthodes ont été appliquées à un jeu de données réelles qui étudie les effets, sur les rythmes circadiens du cortisol, une hormone liée stress. Les résultats montrent que les patients atteints du syndrome de Cushing ont perdu les rythmes circadiens et leurs moyennes à 24 heures sont élevées à de très hauts niveaux. Les patients atteints de dépression majeure possèdent les mêmes formes et phases circadiennes que des sujets normaux. Cependant, leurs niveaux moyens à 24 heures sont élevés et les amplitudes ont été diminuées pour quelques patients.

\section{REFERENCES}

Altman, N. S. (1996). Semi-parametric methods for longitudinal data analysis. In Proceedings of the Biometrics Section, American Statistical Association, 49-57.

Boyar, R. M., Witkin, M., Carruth, A., and Ramsey, J. (1979). Circadian cortisol secretory rhythms in cushing's disease. Journal of Clinical Endocrinology and Metabolism 48, 760-765.

Breiman, L., Friedman, J. H., Olshen, R., and Stone, C. J. (1984). Classification and Regression Trees. New York: Chapman and Hall.

Castro, M., Elias, P. C. L., and Quidute, R. P. (1999). Out-patient screening for Cushing's syndrome: The sensitivity of the combinations of circadian rhythm and overnight dexamethasone suppression salivary cortisol tests. Journal of Clinical Endocrinology and Metabolism 84, 878-882.

Cauter, E. V., Leproult, R., and Kupfer, D. J. (1996). Effects of gender and age on the levels and circadian rhythmicity of plasma cortisol. Journal of Clinical Endocrinology and Metabolism 81, 2468-2473.

Cugini, P., Leone, G., Palma, L. D., Battisti, P., Wilson, D. W., Kawasaki, T., Tamura, K., Halberg, F., and 
Cornelissen, G. (1990). Construction of reference standards for blood pressure 24-h patterns. Statistician 39, 285-299.

Gu, C. (2002). Smoothing Spline ANOVA Models. New York: Springer.

Guardabasso, V., Rodbard, D., and Munson, P. J. (1987). A model-free approach to estimation of relative potency in dose-response curve analysis. American Journal of Physiology 252, 357-364.

Härdle, W. and Marron, J. S. (1990). Semiparametric comparison of regression curves. Annals of Statistics 18, 6389.

Hayes, D. K., Pauly, J. E., and Reiter, R. J. (1990). Chronobiology: Its role in clinical medicine, general biology, and agriculture. In Proceedings of the XIX International Conference of the International Society for Chronobiology, Bethesda, Maryland, June 20-24, 1989. New York: Wiley-Liss.

Ke, C. and Wang, Y. (2001). Semi-parametric nonlinear mixed effects models and their applications (with discussion). Journal of the American Statistical Association 96, 1272-1298.

Kronfol, Z., Nair, M., Zhang, Q., Hill, E., and Brown, M. (1997). Circadian immune measures in healthy volunteers: Relationship to hypothalamic-pituitary-adrenal axis hormones and sympathetic neurotransmitters. Psychosomatic Medicine 59, 42-50.

Kupfer, D. J., Monk, T. H., and Barchas, J. D. (1988). Biological rhythms and mental disorders. New York: Guilford Press.

Lawton, W. H., Sylvestre, E. A., and Maggio, M. S. (1972). Self-modeling nonlinear regression. Technometrics 13, $513-532$.

Lindstrom, M. J. (1995). Self modeling with random shift and scale parameters and a free-knot spline shape function. Statistics in Medicine 14, 2009-2021.

Lindstrom, M. J. and Bates, D. M. (1990). Nonlinear mixed effects models for repeated measures data. Biometrics 46, 673-687.

Liu, J. H., Kazer, R. R., and Rasmussen, D. D. (1987). Characterization of the twenty-four hour secretion patterns of adrenocorticotropin and cortisol in normal wowen and patients with Cushing's disease. Journal of Clinical Endocrinology and Metabolism 64, 1027-1035.

Monk, T. H. (1982). Research methods of chronobiology. In Biological Rhythms, Sleep, and Performance. W. B. Webb (ed), 27-57. New York: Wiley.

Orth, D. N. (1995). Cushing's syndrome. New England Journal of Medicine 32, 791-803.

Pinheiro, J. and Bates, D. M. (2000). Mixed-Effects Models in $S$ and $S$-plus. New York: Springer.

Prins, J. D. and Hecquet, B. (1992). Data processing in chronobiological studies. In Biological Rhythms in Clinical and Laboratory Medicine, Y. Touitou and E. Haus (eds), 90-113. Berlin: Springer.
Raff, H., Raff, J. L., and Findling, J. W. (1998). Late-night salivary cortisol as a screening test for Cushing's syndrome. Journal of Clinical Endocrinology and Metabolism 83, 2681-2686.

Ramsay, J. O. and Li, X. (1998). Curve registration. Journal of the Royal Statistical Society, Series B 60, 351-363.

Redfern, P. H. and Lemmer, B. (1997). Physiology and pharmacology of biological rhythms. Berlin: Springer.

Refetoff, S., Van Cauter, E., Fang, V. S., Laderman, C., Graybela, M. L., and Landau, R. L. (1985). The effect of dexamethasone on the 24-hour profiles of adrenocorticotropin and cortisol in Cushing's syndrome. Journal of Clinical Endocrinology and Metabolism 60, 527-535.

Reilly, T., Atkinson, G., and Waterhouse, J. (1997). Biological Rhythms and Exercise. New York: Oxford.

Stutzle, W., Gasser, T., Molinari, L., Largo, R. H., Prader, A., and Huber, P. (1980). Shape-invariant modeling of human growth. Annals of Human Biology 7, 507-528.

Touitou, Y. and Haus, E. (1992). Biological Rhythms in Clinical and Laboratory Medicine. Berlin: Springer.

Tourniaire, J., Chalendar, D., and Rebbatu, B. (1986). The 24-hour cortisol secretory pattern in Cushing's syndrome. Acta Endocrinology (Copenhagen) 112, 230-237.

Tsaneva, N., Topalova, M., and Beraha, R. (1990). Relation of MESOR to circadian amplitude as a criterion of mental load. In Chronobiology: Its role in clinical medicine, general biology, and agriculture, D. K. Hayes, J. E. Pauly, and R. J. Reiter (eds), 99-103. New York: Wiley.

Wahba, G. (1990). Spline Models for Observational Data. CBMS-NSF Regional Conference Series in Applied Mathematics, Vol. 59. Philadelphia: SIAM

Wang, Y. (1998). Smoothing spline models with correlated random errors. Journal of the American Statistical Association 93, 341-348.

Wang, Y. and Brown, M. B. (1996). A flexible model for human circadian rhythms. Biometrics 52, 588-596.

Wang, Y. and Ke, C. (2002). ASSIST: A Suite of $S$ Plus Functions Implementing Spline Smoothing Techniques. Available at: http://www.pstat.ucsb.edu/ faculty/yuedong/research.

Wang, Y., Guo, W., and Brown, M. B. (2000). Spline smoothing for bivariate data with applications to association between hormones. Statistica Sinica 10, 377-397.

Wever, R. A. (1979). The Circadian System of Man. New York: Springer.

Wong, M. L., Kling, M. A., Munson, et al. (2000). Pronounced and sustained central hypernoradrenergic function in major depression with melancholic features: Relation to hypercortisolism and corticotropin releasing hormone. Proceedings of the National Academy of Sciences USA 97, 325-330.

Received October 2002. Revised April 2003. Accepted May 2003. 